\title{
Efeitos da eletroacupuntura, aquapuntura e farmacopuntura em cadelas anestesiadas com isofluorano e submetidas à ovário-histerectomia
}

\author{
[Effects of electroacupuncture, aquapuncture and farmacopuntura in dogs anesthetized with \\ isoflurane and submitted to ovariohysterectomy] \\ M.O. Taffarel ${ }^{1,5}$, A.E.P. Salgado ${ }^{1}$, E.V. Melo Filho ${ }^{1}$, L.R. Teixeira ${ }^{2,6}$, L.D.C. Fracalossi ${ }^{2,6}$, \\ M.R. Luz ${ }^{3}$, P.M.C. Freitas ${ }^{4 *}$ \\ ${ }^{1}$ Aluna de pós-graduação - Centro de Ciências Agrárias - Universidade Federal do Espírito Santo - CCA-UFES \\ ${ }^{2}$ Aluna de graduação - Universidade Federal do Espírito Santo, ES \\ ${ }^{3}$ Centro de Ciências Agrárias - Universidade Federal do Espírito Santo, ES \\ ${ }^{4}$ Escola de Veterinária - Universidade Federal de Minas Gerais - Belo Horizonte, MG \\ ${ }^{5}$ Bolsista CAPES \\ ${ }^{6}$ Bolsista de Iniciação Científica - FAPES - CCA-UFES
}

\begin{abstract}
RESUMO
Avaliaram-se os efeitos analgésicos da eletroacupuntura, aquapuntura e farmacopuntura com morfina nos acupontos VB41 e TA5 de 24 cadelas hígidas submetidas à ovário-histerectomia. Os animais foram distribuídos em quatro grupos (G) de igual número - GDest, GMorf, GElet e GC - e anestesiados com acepromazina, propofol e isofluorano. Após a estabilização do plano anestésico, os animais do GDest receberam $0,5 \mathrm{~mL}$ de água destilada em cada acuponto; os do GMorf receberam $0,1 \mathrm{mg} / \mathrm{kg}$ de morfina distribuído nos quatro acupontos; os do GElet foram submetidos à eletroacupuntura; e os do GC, acupuntura em pontos sham. Os animais do GC receberam, após o término do procedimento cirúrgico e antes do início da avaliação pelas escalas de dor, $2,0 \mathrm{mg} / \mathrm{kg}$ de tramadol. Foram avaliadas: frequências cardíaca e respiratória, temperatura retal e glicemia. A dor foi avaliada por duas escalas, uma de analogia numérica e outra contagem variável, por três observadores. A avaliação iniciou-se imediatamente após a extubação e foi realizada a cada 15 minutos, durante duas horas. Não houve diferença entre os tratamentos em todas as variáveis. Pode-se concluir que eletroacupuntura, aquapuntura e farmacopuntura com morfina resultaram em analgesia similar ao tramadol no pós-operatório imediato de cadelas submetidas à ovário-histerectomia eletiva.
\end{abstract}

Palavras-chave: cão, analgesia, dor, parâmetros fisiológicos

\begin{abstract}
In order to evaluate the analgesic effects of electro-acupuncture, aquapuncture and morphine pharmacopuncture on VB4land TA5 acupoints, 24 healthy bitches were anesthetized with acepromazine, propofol and isoflurane and underwent ovariohysterectomy. The animals were equally divided into four groups: GDest, GMorf, GElet and CG. After anesthetic stabilization, GDest animals received pure water (0.5ml per acupoint); GMorf received morphine $(0.1 \mathrm{mg} / \mathrm{kg})$; electroacupuncture was performed in GElet animals, and CG animals were submitted to acupuncture on Sham points. The CG animals received $2.0 \mathrm{mg} / \mathrm{kg}$ of tramadol after the end of surgery and before the postoperative evaluation through pain scales. Data recorded were: heart and respiratory rate, rectal temperature and blood glucose. Pain was assessed using two different scales, one using numerical analogy and another using variable counting, both performed by three different professionals. Evaluation started immediately after the animal was extubated and was performed every 15 minutes, for 2 hours. There was no statistical difference between groups in all variables studied. It is concluded that electro-acupuncture, aquapuncture and morphine pharmacopuncture produce analgesia similar to tramadol in the postoperative period of bitches undergoing elective ovariohysterectomy.
\end{abstract}

Keywords: dog, analgesia, pain, physiological parameters

Recebido em 1 de setembro de 2010

Aceito em 4 de julho de 2011

*Autor para correspondência (corresponding author)

E-mail: pcoletto@yahoo.com.br 


\section{INTRODUÇÃO}

A acupuntura é uma técnica da medicina tradicional chinesa (MTC) que utiliza a estimulação de pontos específicos do corpo, chamados acupontos, para o tratamento de enfermidades e analgesia. A ação analgésica promovida por essa técnica decorre da liberação de noradrenalina e da ativação dos receptores $\alpha-2$ agonistas da medula espinhal (Koo et al., 2008), da secreção de ocitocina pelo núcleo supraótico (Yang et al., 2008), da ativação de receptores opioides $\mu$, resultando em liberação de endomorfina-endorfina e receptores $\delta$, liberando encefalina (Zhang et al., 2004; Zhang et al., 2005), além de modulação da atividade dos receptores n-metil-d-aspartato (Wang et al., 2006). Diversas técnicas podem ser utilizadas para estimulação dos pontos de acupuntura, como a eletroacupuntura - que consiste na passagem de corrente elétrica pelo acuponto (Draehmpaehl e Zohmann, 1997) -, a aquapuntura - que se refere à aplicação de água destilada ou solução de cloreto de sódio a $0,9 \%$ e a farmacopuntura - que se utiliza da aplicação de fármacos em doses reduzidas (Luna et al., 2008).

Os opioides, analgésicos utilizados na medicina veterinária, são efetivos no tratamento da dor aguda pós-operatória. O tramadol, um opioide atípico, possui ação central que estimula a liberação de serotonina e a recaptação de noradrenalina, além de possuir afinidade moderada pelo receptor opioide $\mu$ (Raffa et al., 1992). O tramadol foi utilizado com sucesso no tratamento da dor após a ovário-histerectomia em cadelas, pelas vias sistêmica (Mastrocinque e Fantoni, 2003) e epidural (Caldeira et al., 2006), sem promover alterações significativas nos sistemas cardiovascular e respiratório (Mastrocinque e Fantoni, 2003). A morfina, protótipo dos analgésicos opioides, promove analgesia pela afinidade com receptores $\mu$. Contudo, pode promover efeitos adversos, como vômito, liberação de histamina, depressão do centro termorregulatório e depressão respiratória e, em altas doses, bradicardia. A dose em cães varia de 0,2 a $1,0 \mathrm{mg} / \mathrm{kg}$ por via intramuscular ou subcutânea (Pascoe, 2000).

A avaliação da dor em animais é complexa por possuir caráter subjetivo. Dessa forma, estudos têm sido realizados com o objetivo de desenvolver e/ou adaptar escalas para mensuração da dor em diferentes espécies. De acordo com Hardie (2002), a analogia numérica consiste em uma escala numerada com valores de zero - ausência de dor - a 10 - pior dor possível. Para a avaliação da dor pós-operatória em cães, Firth e Haldane (1999) desenvolveram a "Escala de Dor da Universidade de Melbourne", que avalia tanto características fisiológicas como comportamentais, com escore mínimo de zero e máximo de 27 pontos.

Assim, como não há na literatura consultada estudos que comparem aquapuntura, farmacopuntura e eletroacupuntura, este trabalho teve o objetivo de avaliar os efeitos dessas técnicas sobre as características cardiorrespiratórias, o consumo de isofluorano e a analgesia no pós-operatório de cadelas submetidas à ovário-histerectomia ( $\mathrm{OSH})$ eletiva.

\section{MATERIAL E MÉTODOS}

Foram utilizadas 24 cadelas, adultas, hígidas, com peso médio $10,23 \pm 2,60 \mathrm{~kg}$, distribuídas aleatoriamente em quatro grupos de igual número: GDest - aquapuntura com água destilada; GMorf - farmacopuntura com morfina; GElet - eletroacupuntura; e GC animais submetidos à acupuntura em pontos sham.

Após período de restrição hídrica e alimentar de quatro e 12 horas, respectivamente, os animais receberam medicação pré-anestésica acepromazina $\quad 0,1 \mathrm{mg} / \mathrm{kg} \quad \mathrm{IM} \quad-$ e foram submetidos à indução anestésica pelo propofol, por via intravenosa, na dose que possibilitou intubação orotraqueal. $\mathrm{Na}$ manutenção da anestesia, foi utilizado isofluorano em circuito anestésico circular valvular com reinalação parcial de gases, com fluxo diluente de $50 \mathrm{~mL} / \mathrm{kg} / \mathrm{h}$ de oxigênio.

Adquirido o plano anestésico, os animais foram contidos em decúbito dorsal, sobre colchão térmico passivo. Ato contínuo, os acupontos VB41 e TA5 foram localizados anatomicamente de acordo com Draehmpaehl e Zohmann (1997). Nos acupontos dos animais do GDest, foram administrados $0,5 \mathrm{~mL}$ de água destilada em cada ponto; nos do GMorf, administrou-se morfina na 
dose de $0,1 \mathrm{mg} / \mathrm{kg}$ (dose total por animal) diluída em água destilada, quando necessário, até atingir o volume de $0,5 \mathrm{~mL}$ em cada ponto; nos do GElet, foram introduzidas agulhas de acupuntura de $0,18 \times 8,0 \mathrm{~mm}$, em ângulo de $90^{\circ}$ com a pele; e nos do GC, foram introduzidas agulhas idênticas às descritas anteriormente, em acupontos falsos (sham), localizados 10 a 20mm lateralmente aos verdadeiros (Ballegard et al., 1986). Em todos os grupos, o procedimento descrito foi bilateral. No grupo GElet, realizou-se eletroestimulação dos acupontos com corrente alternada, onda tipo quadrada, com frequência de quatro e $100 \mathrm{~Hz}$. No grupo GC, as agulhas foram conectadas ao aparelho de eletroacupuntura, entretanto este permaneceu desligado. Aguardou-se, em todos os grupos, um período de latência de 30 minutos desses procedimentos para o início da cirurgia. Nos animais do GC, ao final da cirurgia e anterior ao início da avaliação pós-operatória, administrou-se tramadol na dose de $2,0 \mathrm{mg} / \mathrm{kg}$, por via intravenosa.

O estudo foi encoberto, ou seja, o anestesista não sabia para qual tratamento o animal se destinava. Realizou-se todo o experimento no período matutino, com o objetivo de minimizar influência do ritmo circadiano sobre o requisito de anestésicos (Vale et al., 1990). A temperatura ambiente do centro cirúrgico foi mantida constante, em $24,6 \pm 1,00^{\circ} \mathrm{C}$ em média.

Durante o procedimento cirúrgico, foram avaliados: temperatura retal (TR frequência respiratória (FR); frequência cardíaca (FC), por avaliação de intervalo R-R em traçado eletrocardiográfico; ocorrência de arritmias, pela avaliação de traçado eletrocardiográfico em eletrocardiógrafo digital; saturação de oxiemoglobina $\left(\mathrm{SpO}_{2}\right)$, obtida por oxímetro de pulso; pressão arterial média (PAM), obtida por método invasivo na artéria femoral com manômetro de mercúrio; glicemia, aferida em glicosímetro portátil; volume total de isofluorano consumido (em $\mathrm{mL}$ ); tempo cirúrgico (em minutos) e volume de vapor inspirado de isofluorano (VIvol\%), conforme Fortis e Jegier (2004).

Durante o pós-operatório, avaliaram-se as FC, FR, TR e glicemia. Para avaliação da dor pósoperatória, foram utilizadas a escala de analogia numérica (EAN) (Holton et al., 1998) e a escala de dor da Universidade de Melbourne
(University Melbourne Pain Scale - UMPS), com valor máximo de 27 pontos.

As variáveis foram aferidas no momento anterior à administração de acepromazina (Mbasal), 10 minutos após a administração da acepromazina (MAcp), após a indução e estabilização anestésica (M0), 30 minutos após o início da estimulação dos acupontos e anterior à incisão de pele (M1), e a cada 10 minutos após M1, durante 60 minutos, coincidindo com os momentos de maior estímulo doloroso - ligadura dos pedículos ovarianos esquerdo (M2) e direito (M3), ligadura do coto uterino (M4), sutura do peritônio (M5) e da pele (M6). No pós-operatório, as variáveis foram aferidas após extubação do animal (Mp0) e a cada 15 minutos, até completar duas horas (Mp15 a Mp120), exceto a glicose, que foi aferida no MBasal, M3, Mp0 e Mp120.

Todos os procedimentos para avaliação da dor foram realizados por três observadores diferentes, que desconheciam o tratamento a que os animais foram submetidos. $O$ resgate analgésico com tramadol foi realizado quando os escores de avaliação da dor chegassem ao valor de cinco pontos na EAN ou nove na UMPS, na observação de, pelo menos, um avaliador. Ao final do experimento, administraram-se enrofloxacina - 5,0mg/kg - por via subcutânea, flunixin meglumine $-1,1 \mathrm{mg} / \mathrm{kg}-\mathrm{e}$ tramadol $2,0 \mathrm{mg} / \mathrm{kg}-$, ambos por via intravenosa.

Utilizou-se o teste de Kruskal-Wallis, para comparação das médias entre momentos de cada tratamento e entre tratamentos em cada momento, com nível de significância de $5 \%$. Para realização das análises, utilizou-se o programa SAEG.

\section{RESULTADOS E DISCUSSÃO}

A escolha dos acupontos utilizados foi baseada nos fundamentos da medicina tradicional chinesa, segundo a qual a dor é resultado de uma condição de excesso ou deficiência de $Q i$ ou de sangue, e a acupuntura regulariza estes estados (Gaynor, 2000). De acordo com Draehmpaehl e Zohmann (1997), a associação dos acupontos TA5 (Waiguan) e VB41 (Zulinqi) é indicada quando se objetiva ação antiálgica, principalmente na região do abdome, já que o VB41 abre o meridiano do vaso da cintura e, 
juntamente com o TA5, regula as laterais do corpo, como a região do ovário.

Neste estudo, optou-se por utilizar para a eletroacupuntura, corrente alternada com frequência de quatro e $100 \mathrm{~Hz}$, pois, de acordo com Han (2004), estímulos de alta $(100 \mathrm{~Hz})$ e baixa $(4 \mathrm{~Hz})$ frequência resultam em melhor analgesia, por liberação de diferentes neuropeptídeos, com menor período de latência e maior duração do efeito analgésico. O estímulo bilateral foi utilizado por promover esses mesmos efeitos (Cassu et al., 2008).

O volume utilizado neste estudo para aquapuntura e farmacopuntura baseou-se na recomendação de Altman (2006). A dose de morfina escolhida, 1/10 da dose convencional de $1,0 \mathrm{mg} / \mathrm{kg}$, foi definida com base em estudo no qual doses de fármacos reduzidas nesta proporção apresentam eficácia semelhante à da dose convencional, quando utilizadas nos acupontos (Luna et al., 2008).

O período de observação pós-operatória está de acordo com o descrito por Draehmpaehl e Zohmann (1997), os quais descreveram que o estímulo de acupontos com alta frequência nas extremidades anteriores e posteriores promove analgesia central apenas durante o período de estímulo. Já o estímulo de baixa frequência promove hipoalgesia gradativa que pode durar até 30 minutos após cessar o estímulo.

O tempo cirúrgico médio dos grupos GDes, GMorf, GElet e GC foi, respectivamente, de $52,03 \pm 0,43 ; \quad 51,16 \pm 2,89 ; \quad 52,33 \pm 1,83$; $52,66 \pm 1,85$, e não se observou diferença estatística entre eles $(\mathrm{P}>0,05)$.

Com relação à TR, não houve diferença entre os grupos em nenhum momento $(\mathrm{P}>0,05)$. Entretanto, observou-se redução significativa entre os momentos nos animais do GDest a partir de M3; nos do GMorf e GC a partir de M2; e nos do GElet a partir de M1. Contudo, os resultados estão dentro dos limites esperados durante um procedimento cirúrgico de cavidade abdominal (Yazbeck, 2002) e foram semelhantes aos encontrados por Pignaton et al. (2007), ao compararem os efeitos da eletroacupuntura e da morfina em gatas submetidas à ovário-histerectomia. A perda de calor é uma complicação frequente no período transoperatório e ocorre por depressão dos mecanismos termostáticos do hipotálamo e depressão metabólica promovida pelos anestésicos (Yazbeck, 2002), associada à exposição do paciente a baixa temperatura da sala cirúrgica (Biazzotto et al., 2006).

Quanto à FR, não houve diferença entre os momentos, exceto no grupo GDest, que apresentou redução significativa em Mp120 (120 minutos após a extubação), quando comparado ao Mbasal. Entretanto, todos os animais apresentaram redução da FR após a administração da acepromazina (MAcp), porém os valores mantiveram-se dentro do intervalo considerado fisiológico para a espécie - 18,50 movimentos por minuto (mpm) para o grupo GDest, 16,90mpm para GMorf, 22,00mpm para GElet, e 20,40mpm para GC, resultados semelhantes aos citados por Gross (2003), ao afirmar que há acentuada redução na FR após a administração de acepromazina em cães.

A média e o erro-padrão do volume de vapor inspirado de isofluorano (VIvol\%)

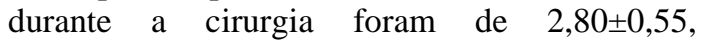
$2,63 \pm 0,35,2,20 \pm 0,25$ e $2,95 \pm 0,38(\mathrm{P}>0,05)$ para os tratamentos GDest, GMorf, GElet e GC, respectivamente. Percentualmente, essas diferenças do VIvol\% foram apenas de 8,1, 10,8 e $25,4 \%$ entre GDest, GMorf e GElet em relação ao GC. Jeong e Nam (2003) demonstraram que a estimulação dos pontos IG4, BP6, E36 e TA8 reduziu a concentração alveolar mínima do isofluorano em cães em 17,5 $\pm 3,1 \%, 21,3 \pm 8,0 \%$, $21,2 \pm 7,5 \%$ e $15,4 \pm 3,1 \%$, respectivamente. A redução do VIvol\% mais acentuada no GElet pode ser atribuída ao efeito analgésico da eletroacupuntura, que resulta em ativação de vias analgésicas opioides e não opioides. A redução do volume inspirado de isofluorano não foi influenciada pelo peso dos animais, pois foi encontrada correlação negativa $(r=-0,1295)$ e não significativa $(\mathrm{P}=0,2731)$ entre as variáveis peso e VIvol\%.

Não se observou diferença $(\mathrm{P}>0,05)$ quanto à $\mathrm{FC}$ entre os grupos e entre momentos (Fig. 1). Estes resultados confirmam os já verificados por outros estudos no homem, ao utilizarem eletroacupuntura em procedimentos cirúrgicos (Kvorning et al., 2003), e em cães submetidos a estímulos nociceptivos (Cassu et al., 2008). Segundo Glowaski (2002), a dor promove 
aumento da FC, o que resulta em maior consumo de anestésico para manter as variáveis fisiológicas estáveis. Neste estudo, o GC apresentou, durante o transcirúrgico, um volume inspirado de isofluorano $25,4 \%$ maior que o GElet para manter os variáveis fisiológicas, indicando, assim, efeito analgésico da estimulação desses acupontos.

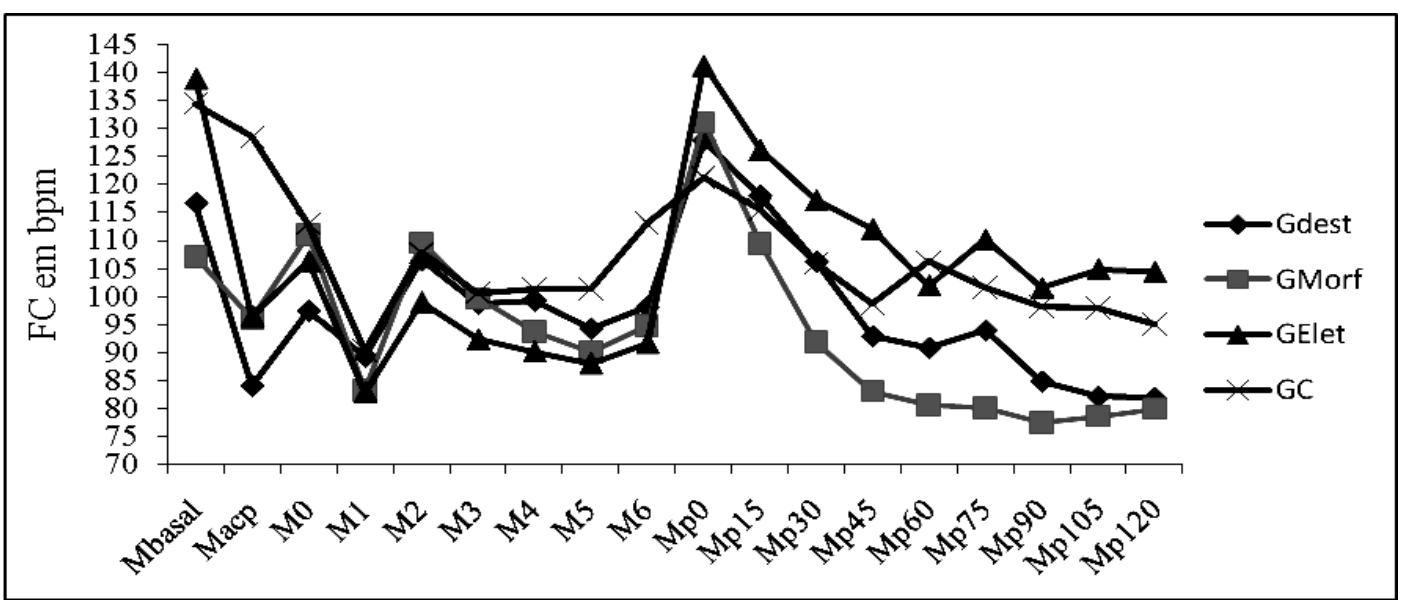

Figura 1. Frequência cardíaca (FC) em cadelas, segundo os tratamentos. GDest: aquapuntura com água destilada; GMorf: farmacopuntura com morfina; GElet: eletroacupuntura; GC: acupuntura em pontos sham.

Com relação à análise eletrocardiográfica, em todos os grupos houve animais que apresentaram bloqueio atrioventricular de primeiro grau (BAV-I) após a administração da acepromazina. No GC, um animal $(16,6 \%)$ apresentou, em MAcp, bloqueio sinoatrial. Segundo Tilley (1992), a acepromazina promove depressão da contratilidade, excitabilidade miocárdica e redução da velocidade de condução do impulso elétrico. Entretanto, não foram observadas complicações decorrentes destas arritmias.

No eletrocardiograma, a onda $\mathrm{T}$ representa a repolarização ventricular e, geralmente, sua amplitude não deve ultrapassar $25 \%$ da amplitude da onda R (Tilley, 1992). O aumento da onda $\mathrm{T}$ durante o procedimento anestésico pode indicar hipóxia de miocárdio e/ ou desequilíbrio eletrolítico. Neste estudo, observou-se que, nos animais do GDest e GC, ocorreu aumento de onda $\mathrm{T}$ em $53,7 \%$ das observações; nos do GMorf, 55\%; e nos do GElet, 33,3\%. Este resultado sugere que a eletroacupuntura promove efeito protetor do miocárdio com relação à hipóxia e assemelha-se aos resultados obtidos por vários autores, que atribuíram este efeito à inibição do excesso de estímulo simpático, diminuindo o consumo de oxigênio (Li et al., 1998; Longhurst, 2007; Lujan et al., 2007).

A pressão arterial média (PAM) manteve-se dentro dos limites normais para a espécie em todos os momentos e grupos (Haskins, 1996). Não houve diferença significativa entre os grupos e momentos (Tab. 1), exceto no GElet, em que, no M5, quando da sutura de parede abdominal, a PAM foi maior do que no GDest. Entretanto, observou-se que em todos os grupos houve redução da PAM no decorrer dos momentos, em razão da diminuição da resistência vascular periférica promovida pelo isofluorano em cães, como demonstrado por Borges et al. (2008). Os resultados deste estudo diferem dos de Kvorning et al. (2003), que descreveram que mulheres submetidas à acupuntura em pontos sham apresentaram aumento significativo da PAM, após incisão abdominal, diferente das submetidas à eletroacupuntura de baixa frequência nos acupontos IG4, E36, BP9, BP6 e F3. No presente estudo, não foi observado aumento da PAM no GC, possivelmente devido ao fato de o anestesista, ao perceber aumento na PAM, ter aumentado a volatilização de isofluorano a fim de evitar sensibilização central pelo estímulo doloroso. 
Taffarel et al.

Tabela 1. Média e erro-padrão da média da pressão arterial média, segundo os tratamentos, durante o procedimento cirúrgico de ovário-histerectomia eletiva em cadelas

\begin{tabular}{ccccccc}
\hline Grupo & M1 & M2 & M3 & M4 & M5 & M6 \\
\hline GDest & $76,16 \pm 5,83$ & $83,50 \pm 5,69$ & $76,16 \pm 4,63$ & $80,33 \pm 6,70$ & $72,66 \pm 4,95 \mathrm{Aa}$ & $74,16 \pm 4,88$ \\
GMorf & $77,50 \pm 5,59$ & $99,33 \pm 8,38$ & $100,66 \pm 7,49$ & $87,00 \pm 8,22$ & $80,33 \pm 4,19 \mathrm{Aab}$ & $74,66 \pm 6,38$ \\
GElet & $80,66 \pm 7,94$ & $99,66 \pm 7,54$ & $103,00 \pm 6,28$ & $95,66 \pm 8,71$ & $93,66 \pm 6,28 \mathrm{Ab}$ & $86,33 \pm 4,76$ \\
GC & $89,66 \pm 4,66$ & $100,66 \pm 7,26$ & $92,83 \pm 6,79$ & $91,83 \pm 2,71$ & $81,33 \pm 2,72 \mathrm{Aab}$ & $76,16 \pm 3,77$ \\
\hline
\end{tabular}

Médias semelhantes entre momentos (M) e entre grupos (G), exceto em M5, em que os valores de GElet foram diferentes dos valores de GDest pelo teste de Kruskal-Wallis $(\mathrm{P}<0,05)$.

GDest: aquapuntura com água destilada; GMorf: farmacopuntura com morfina; GElet: eletroacupuntura; GC: acupuntura em pontos sham.

Para os valores de glicose sanguínea, não houve diferença $(\mathrm{P}>0,05)$ entre os tratamentos e entre momentos (Tab. 2), e os valores mantiveram-se dentro dos padrões de normalidade, os quais variam de 60 a $130 \mathrm{mg} / \mathrm{dL}$ segundo Nelson e Couto (2001), exceto nos animais do GDest, que apresentaram valores significativamente maiores em M6, quando da sutura da pele, em relação aos valores de Mbasal. De acordo com Ko et al. (2000), o aumento da glicemia durante o procedimento cirúrgico pode ser devido à sensibilização nociceptiva de áreas não suprimidas pela anestesia geral, o que provavelmente justifica o aumento observado neste estudo.

Tabela 2. Média e erro-padrão da média da glicose sanguínea segundo os tratamentos, anterior à anestesia (Mbasal) e durante o procedimento cirúrgico de ovário-histerectomia eletiva em cadelas

\begin{tabular}{ccccccc} 
Grupos & MBasal & M0 & M1 & M3 & M6 & Mp120 \\
\hline GDest & $59,3 \pm 4,6 \mathrm{~A}$ & $87,5 \pm 11,6 \mathrm{AB}$ & $97,8 \pm 12,8 \mathrm{AB}$ & $107,6 \pm 15,7 \mathrm{AB}$ & $127,6 \pm 16,3 \mathrm{BC}$ & $73,8 \pm 4,5 \mathrm{AB}$ \\
GMorf & $68,5 \pm 4,0 \mathrm{~A}$ & $79,5 \pm 5,3 \mathrm{~A}$ & $85,0 \pm 8,0 \mathrm{~A}$ & $95,0 \pm 10,1 \mathrm{~A}$ & $101,8 \pm 7,8 \mathrm{~A}$ & $82,1 \pm 11,34 \mathrm{~A}$ \\
GElet & $72,0 \pm 6,7 \mathrm{~A}$ & $89,6 \pm 4,2 \mathrm{~A}$ & $103,8 \pm 14,59 \mathrm{~A}$ & $105,8 \pm 6,9 \mathrm{~A}$ & $118,4 \pm 8,9 \mathrm{~A}$ & $92,8 \pm 9,4 \mathrm{~A}$ \\
GC & $71,3 \pm 5,9 \mathrm{~A}$ & $80,0 \pm 5,8 \mathrm{~A}$ & $86,1 \pm 5,7 \mathrm{~A}$ & $101,5 \pm 4,8 \mathrm{~A}$ & $103,6 \pm 4,5 \mathrm{~A}$ & $75,5 \pm 4,9 \mathrm{~A}$ \\
\hline
\end{tabular}

Médias semelhantes entre momentos (M) e entre grupos (G), exceto em GDest, em que os valores de MBasal foram diferentes dos valores de M6, pelo teste de Kruskal-Wallis $(\mathrm{P}<0,05)$.

GDest: aquapuntura com água destilada; GMorf: farmacopuntura com morfina; GElet: eletroacupuntura; GC: acupuntura em pontos sham.

Mbasal: antes da administração de acepromazina; M0: após a indução e estabilização anestésica; M1: 30 minutos após estimulação dos acupontos; M3: à ligadura do pedículo ovariano direito; M6: à sutura da pele; Mp120: após duas horas da extubação.

Com relação à avaliação pós-operatória, não foi encontrada diferença entre os valores em nenhum dos momentos, nem entre os tratamentos para todas as variáveis. Mastrocinque e Fantoni (2003) também não observaram alterações na FC e FR no pós-operatório de cadelas submetidas à $\mathrm{OSH}$, quando compararam o tramadol e a morfina. Assim, pode-se sugerir que aquapuntura, farmacopuntura e eletroacupuntura promovem efeito semelhante ao tramadol no pósoperatório imediato, quando se considerou a variável frequência cardíaca. Ao avaliar a dor pela EAN e pela escala UMPS, não houve diferença entre os momentos e tratamentos (Tab. 3 e 4). Pela EAN, os animais apresentaram excelente analgesia, pois os escores atribuídos pelos observadores tiveram o valor mínimo de 1,64 e máximo de 2,58. O mesmo fato foi observado pela escala UMPS, na qual o escore mínimo foi de 2,41, e o máximo de 5,01. Lin et al. (2002) não observaram diferenças no escore de avaliação da dor obtido pela escala de analogia visual, no pós-operatório de cirurgia abdominal em mulheres, quando compararam eletroacupuntura e morfina. Assim, neste estudo, verificou-se que a analgesia promovida pela acupuntura nas duas primeiras horas de pósoperatório foi similar à promovida pela administração de tramadol. Essa analgesia deveu-se ao fato de a acupuntura estimular vias opioides para controle da dor, quando há estimulação de acupontos periféricos do corpo, como descrito por Ulett et al. (1998). Além disso, o estímulo dos pontos, neste experimento, por tempo superior a 30 minutos, promoveu esse efeito antiálgico, pois, de acordo com Zhang et al. (2005), este período é suficiente para causar a liberação de endomorfina-endorfina e encefalina. 
Houve resgate analgésico em todos os grupos. No GDest, foi solicitada administração de tramadol em dois animais (33,3\%), aos 15 minutos de pós-operatório (M15) e aos 60 minutos (M60). No GMorf, o resgate analgésico foi solicitado em dois animais no M15, no grupo GElet, em dois animais aos 30 minutos do pósoperatório (M30), e no GC em um animal $(16,6 \%)$ aos 90 minutos (M90). Estes resultados assemelham-se aos de Lin et al. (2002), que relataram solicitação de administração de

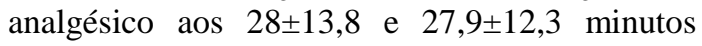
após o término da cirurgia abdominal em mulheres submetidas à eletroacupuntura de alta e baixa frequência, respectivamente. Isso ocorreu, provavelmente, em razão de a resposta analgésica da acupuntura apresentar caráter individual.

Tabela 3. Média e erro-padrão da média de escore da avaliação da dor pela escala de analogia numérica, no pós-operatório de cadelas submetidas à ovário-histerectomia

\begin{tabular}{lcccc}
\hline & GDest & GMorf & GElet & GC \\
Mp0 & $2,27 \pm 0,18$ & $2,22 \pm 0,25$ & $2,16 \pm 0,18$ & $1,66 \pm 0,12$ \\
Mp15 & $2,22 \pm 0,26$ & $2,33 \pm 0,25$ & $2,16 \pm 0,22$ & $1,77 \pm 0,20$ \\
Mp45 & $1,88 \pm 0,14$ & $1,83 \pm 0,20$ & $2,27 \pm 0,31$ & $1,66 \pm 0,12$ \\
Mp60 & $1,72 \pm 0,10$ & $1,94 \pm 0,29$ & $1,94 \pm 0,26$ & $1,55 \pm 0,16$ \\
Mp75 & $1,94 \pm 0,29$ & $1,83 \pm 0,18$ & $1,83 \pm 0,22$ & $1,50 \pm 0,14$ \\
Mp90 & $1,66 \pm 0,08$ & $1,83 \pm 0,20$ & $1,83 \pm 0,22$ & $1,55 \pm 0,14$ \\
Mp105 & $1,72 \pm 0,10$ & $1,66 \pm 0,12$ & $1,66 \pm 0,24$ & $1,50 \pm 0,14$ \\
Mp120 & $1,55 \pm 0,07$ & $1,72 \pm 0,15$ & $1,55 \pm 0,18$ & $1,33 \pm 0,08$ \\
\hline
\end{tabular}

Médias não diferem entre momentos $(\mathrm{M})$ e grupos $(\mathrm{G})$, pelo teste de Kruskal-Wallis $(\mathrm{P}>0,05)$.

M0: após a indução e estabilização anestésica; M1: 30 minutos após estimulação dos acupontos; M3: à ligadura do pedículo ovariano direito; M6: à sutura da pele; Mp120: após duas horas da extubação.

Tabela 4. Média e erro-padrão da média de escore da avaliação da dor pela escala de dor da Universidade de Melbourne, no pós-operatório de cadelas submetidas à ovário-histerectomia

\begin{tabular}{lcccc}
\hline & GDest & GMorf & GElet & GC \\
\hline Mp0 & $3,44 \pm 0,46$ & $3,77 \pm 0,69$ & $3,66 \pm 0,59$ & $3,00 \pm 0,70$ \\
Mp15 & $3,83 \pm 0,68$ & $3,00 \pm 0,52$ & $4,33 \pm 0,68$ & $3,44 \pm 0,93$ \\
Mp30 & $3,66 \pm 0,45$ & $3,05 \pm 0,79$ & $4,27 \pm 0,6$ & $3,55 \pm 0,63$ \\
Mp45 & $3,22 \pm 0,40$ & $3,11 \pm 0,89$ & $3,33 \pm 0,74$ & $3,05 \pm 0,55$ \\
Mp60 & $3,66 \pm 1,01$ & $3,16 \pm 0,68$ & $2,94 \pm 0,59$ & $2,94 \pm 0,80$ \\
Mp75 & $3,50 \pm 1,21$ & $3,11 \pm 0,65$ & $3,11 \pm 0,85$ & $2,89 \pm 0,77$ \\
Mp90 & $3,11 \pm 0,69$ & $2,61 \pm 0,56$ & $2,11 \pm 0,72$ & $2,72 \pm 0,77$ \\
Mp105 & $2,66 \pm 0,88$ & $2,55 \pm 0,58$ & $1,83 \pm 0,58$ & $2,16 \pm 0,60$ \\
Mp120 & $2,61 \pm 0,45$ & $2,33 \pm 0,90$ & $2,27 \pm 0,89$ & $1,83 \pm 0,68$ \\
\hline
\end{tabular}

Médias não diferem entre momentos (M) e grupos (G), pelo teste de Kruskal-Wallis ( $\mathrm{P}>0,05)$.

M0: após a indução e estabilização anestésica; M1: 30 minutos após estimulação dos acupontos; M3: à ligadura do pedículo ovariano direito; M6: à sutura da pele; Mp120: após duas horas da extubação.

\section{CONCLUSÕES}

A eletroacupuntura nos pontos TA5 e VB41 promove estabilidade cardiorrespiratória e reduz o volume de vapor inspirado de isofluorano em cadelas submetidas à ovário-histerectomia. Dentre as técnicas estudadas, a eletroacupuntura mostra-se mais vantajosa em relação às demais na redução do volume de vapor inspirado de isofluorano e na estabilidade cardíaca. As técnicas estudadas promovem analgesia semelhante ao tramadol no pós-operatório imediato de cadelas submetidas à ováriohisterectomia. 


\section{REFERÊNCIAS}

ALTMAN, S. Técnicas e instrumentação. In: SCHOEN, A.M. Acupuntura veterinária: da arte antiga à medicina moderna. São Paulo: Roca, 2006. p.91-108.

BALLEGARD, S.; JENSEN G.; PEDERSEN F. et al. Acupuncture in severe stable angina pectoris: a randomized trial. Acta Med. Scand., v.220, p.307-313, 1986.

BIAZZOTTO, C.B.; BRUDNIEWSK, M.; SCHMIDT, A.P. et al. Hipotermia no período perioperatório. Rev. Bras. Anestesiol., v.56, p.89106, 2006.

BORGES, P.A.; NUNES, N.; BARBOSA, V.F. et al. Variáveis cardiorrespiratórias, índice biespectral e recuperação anestésica em cães anestesiados pelo isofluorano, tratados ou não com tramadol. Arq. Bras. Med. Vet. Zootec., v.60, p.613-619, 2008.

CALDEIRA, F.M.C.; OLIVEIRA, H.P.; MELO, E.G. et al. Cortisol sérico e glicemia em cadelas tratadas com tramadol e submetidas à ováriohisterectomia. Cienc. Rural., v.36, p.155-160, 2006

CASSU, R.N.; LUNA, S.P.L.; CLARK, R.M.O. et al. Electroacupuncture analgesia in dogs: is there a difference between uni- and bi-lateral stimulation? Vet. Anaesth. Analg., v.35, p.52-61, 2008.

DRAEHMPAEHL, D.; ZOHMANN, A. Acupuntura no cão e no gato: princípios básicos e prática científica. São Paulo: Roca, 1997. 254p.

FIRTH, A.M.; HALDANE, S.L. Development of scale to evaluate postoperative pain in dogs. $J$. Am. Vet. Med. Assoc., v.214, p.651-659, 1999.

FORTIS, E.A.F.; JEGIER, M.A. Física e anestesia inalatória. In: MANICA, J. Anestesiologia. Princípios e técnicas. 3. ed. Porto Alegre: Artmed, 2004. p.185-212.

GAYNOR, J.S. Acupuncture for management of pain. Vet. Clin. N. Am.: Small Anim. Pract.,v.30, p.875-884, 2000.

GLOWASKI, M.M. Analgesia in critical care. Vet. Clin. N. Am.: Small Anim. Pract., v.32, p.1127-1144, 2002.
GROSS, K.R. Tranquilizantes, agonistas $\alpha_{2-}$ adrenérgicos e agentes relacionados. In: ADAMS, H.R. (Ed). Farmacologia e terapêutica em veterinária. Rio de Janeiro: GuanabaraKoogan, 2003. p.249-284.

HAN, J.S. Acupuncture and endorphins. Neurosc. Lett., v.361, p.258-261, 2004.

HARDIE, E.M. Reconhecimento do comportamento doloroso em animais. In: HELLEBREKERS, L.J. Dor em animais. São Paulo: Manole, 2002. p. 49-68.

HASKINS, S.C. Monitoring the anesthetized patient. In: THURMON, J.C.;

TRANQULI, W.J.; BENSON, G.J. Lumb \& Jone's veterinary anesthesia. 3.ed. Baltimore: Lippincott Williams e Wilkins, 1996. p.409-424.

HOLTON, L.L.; SCOTT, E.M.; NOLAN, A.M. et al. Comparison of three methods used for assessment of pain in dogs. J. Am. Vet. Med. Assoc., v.212, p.61-66, 1998.

JEONG, S. M.; NAM, T.C. Effect of eletroacupuncture on minimum alveolar concentration of isofluorane in dogs. J. Vet. Med. Sci., v.65, p.145-147, 2003.

KO, J.C.; MANDSAGER, R.E.; LANGE, D.N. et al. Cardiorespiratory responses and plasma cortisol concentrations in dogs treated with medetomidine before undergoing ovariohysterectomy. J. Am. Vet. Med. Assoc., v.217, p.509-514, 2000.

KOO, S.T.; LIM, K.S.; CHUNG, K. et al. Electroacupuncture-induced analgesia in a rat model of ankle sprain pain is mediated by spinal alpha-adrenoceptors. Pain, v.135, p.11-19, 2008.

KVORNING, N.; CHRISTIANSSON, C.; BESKOW, A. et al. Acupuncture fails to reduce but increases anaesthetic gas required to prevent movement in response to surgical incision. Acta Anaesth. Scand., v.47, p.818-822, 2003.

LI, P.; PITSILLIDES, K.F.; RENDIG, S.V. et al. Reversal of reflex-induced myocardial ischemia by median nerve stimulation: a feline model of electroacupuncture. Circulation, v.97, p.11861194, 1998. 
LIN, J.G; LO, M.W.; WEN, Y.R. et al. The effect of high and low frequency electroacupuncture in pain after lower abdominal surgery. Pain, v.99, p.509-514, 2002.

LONGHURST, J.C. Electroacupuncture treatment of arrhythmias in myocardial ischemia. Am. J. Physiol. Heart Circ. Physiol., v.292, p.H2032-H2034, 2007.

LUJAN, H.L; KRAMER, V.J.; DICARLO, S.E. Electroacupuncture decreases the susceptibility to ventricular tachycardia in conscious rats by reducing cardiac metabolic demand. Am. J. Physiol. Heart Circ. Physiol., v.292, p.H2550H2555, 2007.

LUNA, S.P.L.; ANGELI, A.L.; FERREIRA, C.L. et al. Comparison of pharmacopuncture, aquapuncture and acepromazine for sedation of horses. E-Cam, v.5, p.267-272, 2008.

MASTROCINQUE, S.; FANTONI, D.T. A comparison of preoperative tramadol and morphine for the control of early postoperative pain in canine ovariohysterectomy. Vet. Anaesth. Analg., v.30, p.220-228, 2003.

NELSON, R.W.; COUTO, C.G. Distúrbios do pâncreas endócrino. In: Medicina interna de pequenos animais. Rio de Janeiro: Guanabara-Koogan, 2001. p.580-609.

PASCOE, P.J. Opioid analgesics. Vet. Clin. N. Am.: Small Anim. Pract., v.30, p.757-772, 2000.

PIGNATON, W.; SIMÕES, J.R.; ZACCHÉ, E. et al. Efeito da eletroacupuntura e da morfina sobre parâmetros cardiorrespiratórios e consumo de anestésico em gatas submetidas à ovariosalpingohisterectomia. Acta Sci. Vet., v.35, Supl. 2, p. 331-332, 2007.
RAFFA, R.B.; FRIDERICHS, E.; REIMANN, W. et al. Opioid and nonopioid components independently contribute to the mechanism of action of tramadol, an 'atypical' opioid analgesic. J. Pharmacol. Exp. Ther., v.260, p.275-85, 1992.

TILLEY, L.P. Essentials of canine and feline eletrocardiography. 3. ed. Philadelphia: Lea \& Febiger, 1992. 470p.

ULETT, G.A.; HAN, S.; HAN, J. Electroacupuncture: mechanisms and clinical application. Biol. Psych., v.44, p.129-138, 1998.

VALE, N.B.; VALE, L.F.B.; DELFINO, J. Osciladores e sincronizadores na anestesia local. Rev. Bras. Anestesiol., v.40, p.39-47, 1990.

WANG, C.; ZHANG, Y.; DAI, J. et al. Electroacupuncture (EA) modulates the expression of NMDA receptors in primary sensory neurons in relation to hyperalgesia in rats. Brain Res., v.1120, p.46-53, 2006.

YANG, J.; YANG, Y.; CHEN, J.M. et al. Effect of hypothalamic supraoptic nucleus on acupuncture analgesia in the rat. Brain Res. Bull., v.75, p.681-686, 2008.

YAZBECK, K.V.B. Hipotermia. In: FANTONI, D.T.; CORTOPASSI, S.R.G. Anestesia em cães e gatos. São Paulo: Roca, 2002. p. 373 -380.

ZHANG, R.X.; LAO, L.; WANG, L. et al. Involvement of opioid receptors in electroacupuncture-produced anti-hyperalgesia in rats with peripheral inflammation. Brain Res., v.1020, p.12-17, 2004.

ZHANG, R.X.; WANG, L.; LIU, B. et al. Mu opioid receptor-containing neurons mediate electroacupuncture-produced anti-hyperalgesia in rats with hind paw inflammation. Brain Res., v.1048, p.235-240, 2005. 